\title{
Predictors of axillary node response in node-positive patients undergoing neoadjuvant chemotherapy for breast cancer
}

\author{
Farah Ladak, MD, MPH \\ Natalie Chua, MD \\ David Lesniak, MD \\ Sunita Ghosh, PhD \\ Ericka Wiebe, MD \\ Walter Yakimetz, MD \\ Nikoo Rajaee, MD, MHE \\ David Olson, MD \\ Lashan Peiris, MBBS
}

Presented at the American Society of Breast Surgeons 20th Annual Meeting, Apr. 30May 5, 2019, Dallas, Tex., and the 2019 Canadian Breast Surgery Research Forum, Canadian Breast Cancer Symposium 2019, June 13, 2019, Toronto, Ont.

Accepted Mar. 3, 2021

\section{Correspondence to:}

L. Peiris

Misericordia Hospital

166 Meadowlark Health Centre

156 St and 87 Ave

Edmonton AB T5R 5W9

peiris@ualberta.ca

Cite as: Can J Surg 2022 February 8; 65(1). doi: $10.1503 /$ cjs. 012920

\begin{abstract}
Background: The ability to accurately predict which patients will achieve a pathologic complete response (pCR) after neoadjuvant chemotherapy could help identify those who could safely be spared the potential morbidity of axillary lymph node dissection. We performed a retrospective analysis of a cohort of clinically node-positive patients managed with neoadjuvant chemotherapy with the goal of identifying predictors of axillary pCR.
\end{abstract}

Methods: Eligible patients were aged 18 years or older, had clinical T1-T4, N1-N3, M0 breast cancer and received neoadjuvant chemotherapy followed by surgical axillary lymph node staging between 2001 and 2017 at Misericordia Hospital, Edmonton, Alberta. Patient data, including tumour characteristics, details of neoadjuvant chemotherapy, imaging results before and after neoadjuvant chemotherapy, and final pathologic analysis, were collected from the appropriate provincial electronic data repositories. We summarized the data using descriptive statistics. We characterized associations between clinical/tumour characteristics and pCR using univariate and multivariate regression analysis.

Results: Of the 323 patients included in the study, 130 (40.2\%) achieved axillary pCR. Absence of residual disease in the breast was associated with axillary pCR (odds ratio $6.74,95 \%$ confidence interval 2.89-15.67). HER2-positive, triple-negative and ER-positive/PR-negative/HER2-negative tumours were significantly associated with a pCR on univariate analysis; the association trended toward significance on multivariate analysis.

Conclusion: Our findings support the routine use of neoadjuvant chemotherapy and sentinel lymph node biopsy in patients with an absence of residual disease in the breast, and potentially in those with HER2-positive or triple-negative subtypes, and highlight the ER-positive/PR-negative biomarker subtype as a potential predictor of nodal response.

Contexte : La capacité de prédire avec précision quelles patientes obtiendront une réponse pathologique complète $(\mathrm{RpC})$ après une chimiothérapie néoadjuvante pourrait faciliter l'identification de celles chez qui on pourrait sécuritairement éviter la morbidité potentielle associée à l'exérèse des ganglions lymphatiques axillaires. Nous avons procédé à une analyse rétrospective sur une cohorte de patientes traitées par chimiothérapie néoadjuvante dont les ganglions étaient cliniquement positifs, dans le but de dégager les prédicteurs d'une $\mathrm{RpC}$ axillaire.

Méthodes : Les patientes admissibles avaient 18 ans ou plus, présentaient un cancer du sein clinique M0 T1-T4, N1-N3 et avaient reçu une chimiothérapie néoadjuvante suivie d'une stadification chirurgicale des ganglions lymphatiques axillaires entre 2001 et 2017 à l'Hôpital Misecordia d'Edmonton, en Alberta. Les données sur les patientes, y compris les caractéristiques des tumeurs, les détails de la chimiothérapie néoadjuvante, les résultats de l'imagerie avant et après la chimiothérapie néoadjuvante et l'analyse anatomopathologique finale ont été recueillis à partir des registres de données électroniques provinciaux appropriés. Nous avons résumé les données sous forme de statistiques descriptives. Nous avons dégagé les liens entre les caractéristiques cliniques/tumorales et la $\mathrm{RpC}$ à l'aide d'analyses de régression univariées et multivariées.

Résultats : Parmi les 323 patientes incluses dans l'étude, 130 (40,2\%) avaient obtenu une $\mathrm{RpC}$ axillaire. L'absence de maladie résiduelle dans le sein a été associée à une $\mathrm{RpC}$ axillaire (rapport des cotes 6,74, intervalle de confiance de $95 \%$ 2,89-15,67). Les tumeurs HER2-positives, triples négatives et ER-positives/PR-négatives/HER2négatives ont été significativement associées à une $\mathrm{RpC}$ à l'analyse univariée; à l'analyse multivariée, l'association tendait à être significative. 


\begin{abstract}
Conclusion : Nos conclusions appuient l'utilisation de routine de la chimiothérapie néoadjuvante et la biopsie des ganglions sentinelles chez les patientes en l'absence de maladie résiduelle dans le sein, et potentiellement chez celles qui présentent les soustypes HER2-positif ou triple négatif, et elles évoquent le sous-type de biomarqueur ER-positif/PR-négatif comme prédicteur potentiel d'une réponse ganglionnaire.
\end{abstract}

A xillary lymph node status is an important determinant of long-term survival in patients with breast cancer and is used to guide local, regional and systemic treatment. ${ }^{1,2}$ In patients with clinically node-negative disease, sentinel lymph node biopsy (SLNB) has replaced axillary lymph node dissection (ALND) for axillary staging. ${ }^{3-9}$ In patients with clinically node-positive disease, the appropriateness of SLNB remains contentious, particularly after neoadjuvant chemotherapy, where fibrosis is thought to disrupt normal lymphatic flow.

The perceived higher false-negative rate of SLNB after neoadjuvant chemotherapy in node-positive patients is considered a major barrier to using SLNB to surgically stage the axilla after preoperative treatment. ${ }^{10}$ This topic has been investigated in 3 large prospective trials: ACOSOG Z1071 (American College of Surgeons Oncology Group Z1071 clinical trial), ${ }^{11}$ SN FNAC (Sentinel Node Biopsy Following Neoadjuvant Chemotherapy trial) ${ }^{12}$ and SENTINA (SENTinel NeoAdjuvant study). ${ }^{13}$ The overall rate of sentinel node detection was lower than expected in these trials ( $80 \%-87 \%)$, and the false-negative rates were higher than the prespecified threshold of $10 \%$ in 2 of the studies. ${ }^{11,13}$ However, the false-negative rates could be significantly lowered if more than 2 sentinel nodes were removed and if both radioactive tracer and blue dye were used. Furthermore, targeted axillary lymph node dissection, removing the previously clipped node, reduced the false-negative rate of SLNB in this population to around $2 \% .^{14}$

Despite these advances, national guidelines on the use of SLNB after neoadjuvant chemotherapy are lacking. The Canadian Consortium for Locally Advanced Breast Cancer highlighted the latest evidence surrounding the optimal use of neoadjuvant systemic therapy for locally advanced breast cancer, ${ }^{15}$ which favoured SLNB for the purpose of axillary staging as long as ACOSOG Z1071, SENTINA and SN FNAC trial criteria were met. Despite this, many surgeons remain apprehensive about performing SLNB after neoadjuvant chemotherapy.

Neoadjuvant chemotherapy was initially introduced with the goal of downstaging patients with inoperable locally advanced breast cancer. ${ }^{16,17}$ However, it is increasingly being used for earlier-stage disease. A pathologic complete response (pCR) has been observed in 33\%-37\% of women undergoing neoadjuvant chemotherapy for T1-T2 disease, with complete nodal eradication in up to $50 \%$ of women. ${ }^{11,18}$ At present, consensus guidelines support the use of neoadjuvant chemotherapy in patients with operable breast cancer with human epidermal growth factor receptor
2 (HER2)-positive disease or triple-negative breast cancer (TNBC) if $\mathrm{T}$ is 2 or higher, or $\mathrm{N}$ is 1 or higher; if the primary tumour is large relative to breast size in patients desiring breast conservation; and in patients with node-positive disease that has a reasonably high likelihood of becoming node-negative. Locally, ALND after neoadjuvant chemotherapy remains the standard of care for clinically nodepositive women, regardless of axillary response.

Although dedicated axillary imaging with ultrasonography or magnetic resonance imaging may go some way to predicting nodal status after neoadjuvant chemotherapy, studies have consistently shown that patients with HER2positive disease or TNBC have the highest probability of achieving pCR after neoadjuvant chemotherapy (up to $63 \%$ for hormone-receptor-negative/HER2-positive patients receiving HER2-targeted therapy, and 37\% for those with TNBC) ${ }^{15,16}$ However, additional histopathologic markers of axillary complete response have been hard to identify.

The ability to predict which patients will achieve pCR can ensure timely receipt of neoadjuvant chemotherapy, while those predicted to be nonresponders can forgo neoadjuvant chemotherapy and proceed directly to ALND. ${ }^{19,20}$ We performed a retrospective analysis of a cohort of clinically node-positive patients managed with neoadjuvant chemotherapy with the goal of identifying predictors of axillary pCR.

\section{Methods}

\section{Study population}

Eligible patients were aged 18 years or older, had clinical T1-T4, N1-N3, M0 breast cancer and received neoadjuvant chemotherapy followed by surgical axillary lymph node staging between 2001 and 2017 at Misericordia Hospital, Edmonton, Alberta (Figure 1). In our institution, axillary ultrasonography is performed in the investigation of patients with BI-RADS 4-5 lesions. Hence, patients who had negative axillae on physical examination but were deemed node-positive after axillary ultrasonography and node biopsy were also included in our study, in keeping with the American foint Committee on Cancer Staging Manual. ${ }^{21}$ We excluded patients with recurrence, those with a history of prior axillary surgery (removal of $\geq 1$ axillary nodes) and those with inflammatory breast cancer (in whom mandatory ALND is recommended, irrespective of their response to neoadjuvant chemotherapy, owing to the well-established inaccuracy 


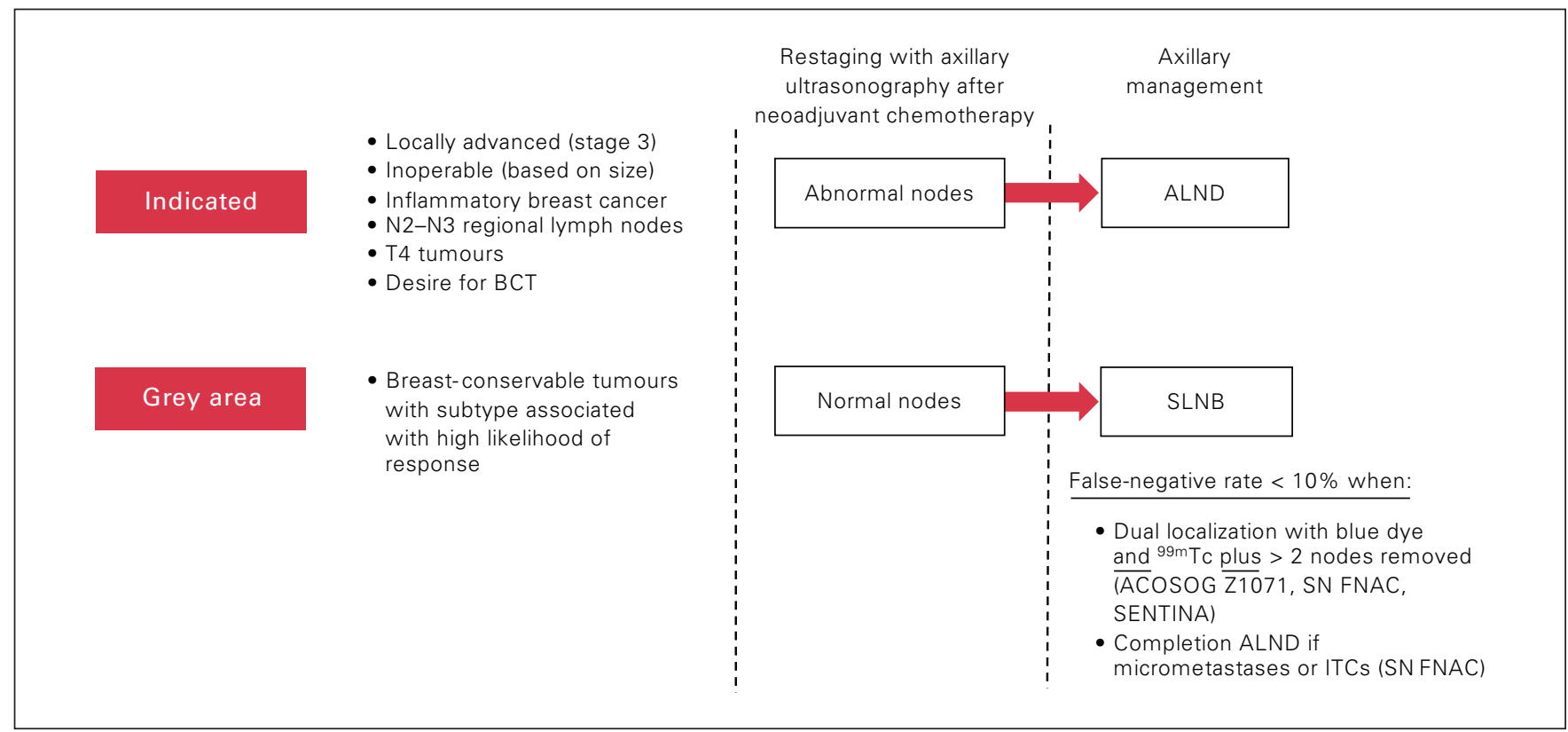

Fig. 1. Current management of clinically node-positive breast cancer. ACOSOG Z1071 = American College of Surgeons Oncology Group Z1071 clinical trial ${ }^{11}$; ALND = axillary lymph node dissection; BCT = breast-conserving therapy; ITC = isolated tumour cell; SENTINA = SENTinel NeoAdjuvant study ${ }^{13} ;$ SLNB = sentinel lymph node biopsy; SN FNAC = Sentinel Node Biopsy Following Neoadjuvant Chemotherapy trial ${ }^{12} ;{ }^{99 m} \mathrm{Tc}=$ technetium $-99 \mathrm{~m}$.

of SLNB with this particular pathophysiology). ${ }^{22} \mathrm{We}$ defined clinical tumour and nodal category according to the American Joint Committee on Cancer breast cancer staging system.

\section{Neoadjuvant chemotherapy}

Indications for neoadjuvant chemotherapy included inoperable or locally advanced breast cancer, inflammatory breast cancer and, in selected cases, early-stage disease to facilitate breast-conservation surgery. Neoadjuvant chemotherapy regimens were administered in accordance with the provincial guidelines. ${ }^{23}$ The balance of the regimens included 5-fluorouracil, epirubicin and cyclophosphamide (FEC), or doxorubicin and cyclophosphamide (AC) for 3-4 cycles, followed by a taxane (generally docetaxel) for 3-4 cycles. Another commonly used regimen was docetaxel, doxorubicin and cyclophosphamide (TAC) for 6 cycles. Patients with HER2-positive disease received FEC or AC for 3-4 cycles, followed by docetaxel and trastuzumab for 3-4 cycles, or docetaxel, carboplatin and trastuzumab for 6 cycles; trastuzumab was continued for 1 year postoperatively in these patients. Regimens were modified according to patient comorbidities. Adjuvant radiotherapy was individualized according to local protocols and multidisciplinary tumour board assessment.

\section{Data collection}

After obtaining Health Research Ethics Board of Alberta ethics approval for this study (HREBA.CC-17-0634), we collected patient data from the appropriate provincial electronic data repositories (Synoptec, Aria or IMPAX). Data collected included patient demographic characteristics, tumour characteristics, details of neoadjuvant chemotherapy, imaging results before and after neoadjuvant chemotherapy, and final pathologic analysis, including number of nodes harvested, number of positive nodes and residual tumour size.

\section{Outcome of interest}

Our primary outcome of interest was axillary pCR, defined as no evidence of residual invasive tumour in the axillary lymph nodes, after neoadjuvant chemotherapy. Patients with micrometastases or isolated tumour cells on pathologic examination after neoadjuvant chemotherapy were considered to have residual node-positive disease.

\section{Statistical analysis}

We used descriptive statistics and frequency tabulation to summarize the data. We reported mean and standard deviation for continuous variables, and frequency and proportion for categoric variables.

We characterized associations between clinical and tumour characteristics and pCR using $\chi^{2}$ tests for categoric variables. We used the Fisher exact test when the cell frequency was less than 5 . We used the MannWhitney test to compare the median for nonnormally distributed continuous variables. We used purposeful selection, employing a combination of variables found to 
be statistically significant in the univariate analysis and factors with established clinical relevance, to a create a multivariate regression model. The final multivariate model comprised clinically and statistically relevant variables. We estimated discrimination of the model using area under the curve of the receiver-operator curve. All statistical analyses were performed with SPSS version 25 (IBM Corp.), and a $p$ value $<0.05$ was considered statistically significant.

\section{Results}

Between October 2001 and November 2017, 517 patients underwent axillary surgery after neoadjuvant chemotherapy for clinically node-positive breast cancer at our institution. Of those, 195 were excluded based on the presence of recurrent disease, missing information or prior axillary lymph node surgery, leaving 323 patients for analysis.

Patient clinicopathologic characteristics by pCR status are presented in Table 1 . The mean patient age was 63 (range 49-74) years, and the median tumour size (recorded as the greatest dimension measured) was $4.14 \mathrm{~cm}$ (range $0.6-11.6 \mathrm{~cm}$ ). The majority of patients $(276$ [85.4\%]) had invasive ductal carcinoma, $16(5.0 \%)$ had invasive lobular cancer, and 9 (2.8\%) had mixed ductal/ lobular cancer; information on histologic type was missing in 22 cases [6.8\%]). Tumour grade and receptor subtype are shown in Table 1.

\section{Neoadjuvant treatment and axillary node dissection}

Axillary imaging results were available for 217 patients, of whom $183(84.3 \%)$ were documented as having an abnormal axilla. Of the $217,112(51.6 \%)$ had more than 1 abnormal node, and 105 (48.4\%) had 1 suspicious node. Preoperative imaging of the breast showed an average reduction in tumour size of $54 \%$ after neoadjuvant chemotherapy. Although axillary imaging was not performed routinely after neoadjuvant chemotherapy, 72 (39.3\%) of 183 patients who were initially documented as having an abnormal axilla showed no evidence of axillary disease on postoperative pathologic examination. Of the 34 patients with a radiographically abnormal axilla, $12(35 \%)$ had no pathologic evidence of nodal disease, and $22(65 \%)$ had $1-19$ (mean 4.4$)$ positive nodes. The average harvest was 13.4 (range 1-41) nodes. Of the 323 patients, $130(40.2 \%)$ achieved a pCR within the axilla, and 125 patients $(38.7 \%)$ achieved a pCR within the breast.

Receptor subtype was highly predictive of $\mathrm{pCR}$ on univariate analysis, but the association did not reach statistical significance on multivariate analysis. Patients with HER2-positive disease had the highest odds of pCR (odds ratio [OR] 5.04, 95\% confidence interval [CI] 2.729.33), followed by those with TNBC (OR 3.82, 95\% CI 1.89-7.70) and those with estrogen receptor (ER)positive/progesterone receptor (PR)-negative/HER2negative disease (OR 3.42, 95\% CI 1.42-8.22) (Table 2).

\begin{tabular}{|c|c|c|c|}
\hline \multirow[b]{2}{*}{ Characteristic } & \multicolumn{3}{|c|}{ No. (\%) of patients* } \\
\hline & $\begin{array}{l}\text { Overall } \\
n=323\end{array}$ & $\begin{array}{c}\text { No pathologic } \\
\text { complete responset } \\
n=196\end{array}$ & $\begin{array}{c}\text { Pathologic complete } \\
\text { responset } \\
n=127\end{array}$ \\
\hline Tumour size, median (range), $\mathrm{cm}$ & $4.14(0.6-11.6)$ & $4.27(0.6-11.6)$ & $3.94(0.8-10.4)$ \\
\hline \multicolumn{4}{|l|}{ Histologic type } \\
\hline Ductal & $276(85.4)$ & $169(61.2)$ & 107 (38.8) \\
\hline Lobular & $16(5.0)$ & $14(87.5)$ & $2(12.5)$ \\
\hline Mixed ductal/lobular & $9(2.8)$ & $9(100.0)$ & $0(0.0)$ \\
\hline Missing & $22(6.8)$ & $4(18.2)$ & $18(81.8)$ \\
\hline \multicolumn{4}{|l|}{ Receptor subtype } \\
\hline $\mathrm{ER}+/ \mathrm{PR}+/ \mathrm{HER} 2-$ & $104(32.2)$ & $85(81.7)$ & 19 (18.3) \\
\hline ER+/PR-/HER2- & $30(9.3)$ & $17(56.7)$ & $13(43.3)$ \\
\hline HER2+ & $117(36.2)$ & $55(47.0)$ & $62(53.0)$ \\
\hline Triple-negative & 63 (19.5) & $34(54.0)$ & $29(46.0)$ \\
\hline Missing & $9(2.8)$ & $5(55.6)$ & $4(44.4)$ \\
\hline \multicolumn{4}{|l|}{ Tumour grade } \\
\hline $1-2$ & $118(36.5)$ & 87 (66.9) & $31(26.3)$ \\
\hline 3 & $178(55.1)$ & $101(58.7)$ & 77 (43.3) \\
\hline Missing & $27(8.4)$ & $8(29.6)$ & 19 (70.4) \\
\hline
\end{tabular}




\begin{tabular}{|c|c|c|}
\hline \multirow[b]{2}{*}{ Predictor } & \multicolumn{2}{|c|}{ OR (95\% Cl) } \\
\hline & Univariate analysis & Multivariate analysis \\
\hline \multicolumn{3}{|l|}{ Histologic type } \\
\hline Lobular (Ref) & 1 & 1 \\
\hline Ductal & 4.43 (0.99-19.89) & $2.16(0.18-26.15)$ \\
\hline Mixed ductal/lobular & - $^{*}$ & - $^{*}$ \\
\hline \multicolumn{3}{|l|}{ Receptor subtype } \\
\hline ER+/PR+/HER2- (Ref) & 1 & 1 \\
\hline ER+/PR-/HER2- & $3.42(1.42-8.22)$ & $2.16(0.60-7.70)$ \\
\hline HER2+ & $5.04(2.72-9.33)$ & $2.41(0.90-6.48)$ \\
\hline Triple-negative & $3.82(1.89-7.70)$ & $2.39(0.88-6.54)$ \\
\hline \multicolumn{3}{|l|}{ Tumour grade } \\
\hline Low (1-2) (Ref) & 1 & 1 \\
\hline High (3) & $2.11(1.27-3.51)$ & $1.78(0.80-3.94)$ \\
\hline \multicolumn{3}{|l|}{ Nodal density } \\
\hline Multiple (Ref) & 1 & - \\
\hline Single & $1.11(0.61-2.03)$ & - \\
\hline \multicolumn{3}{|l|}{ Residual tumour } \\
\hline Yes (Ref) & 1 & 1 \\
\hline No & $7.28(4.31-12.29)$ & $6.74(2.89-15.67)$ \\
\hline Tumour size & $0.93(0.81-1.06)$ & $0.91(0.76-1.08)$ \\
\hline
\end{tabular}

Patients with high-grade (grade 3) tumours were more likely to achieve pCR than those with non-high-grade (grade 1-2) tumours (OR 2.11, 95\% CI 1.27-3.51). Although tumour grade was highly predictive of pCR on univariate analysis, it failed to reach statistical significance on multivariate analysis. Absence of residual tumour on postoperative pathologic examination was highly predictive of a concurrent axillary pCR (OR 7.28, 95\% CI 4.31$12.29)$ on both univariate and multivariate analysis. Tumour histologic type (ductal v. lobular/mixed) fell just short of reaching statistical significance on univariate analysis $(p=0.05$ ), and imaging nodal density (multiple $\mathrm{v}$. single nodes on ultrasonography) was not found to be predictive of pCR.

With the exception of residual tumour burden (OR $6.74,95 \%$ CI 2.89-15.67), independent predictors of pCR identified on univariate analysis were no longer statistically significant on multivariate analysis, although there remained a trend toward higher odds of a pCR among patients depending on their biomarkers (HER2positive, TNBC and ER-positive/PR-negative/HER2negative receptor subtypes), tumour grade and tumour subtype.

\section{Discussion}

In this single-centre retrospective cohort of 323 patients with node-positive breast cancer who underwent axillary surgery after neoadjuvant chemotherapy, $40 \%$ of patients achieved axillary pCR, with the highest rates among women with smaller breast tumours at diagnosis and those with no residual breast tumour after neoadjuvant treatment. Among hormone receptor subtypes, HER2-positive and TNBC had the highest rates of axillary pCR. Patients with ER-positive/PR-negative tumours were almost as likely to achieve pCR as those with triple-negative tumours, a finding that has not been widely described in the literature.

The role of neoadjuvant chemotherapy is expanding as we move toward surgical de-escalation and individualized management plans for patients with breast cancer. At present, neoadjuvant chemotherapy provides an in vivo assessment of treatment response, with an opportunity to switch regimens in nonresponders, and can downstage tumours to breast-conserving treatment, thereby minimizing disfigurement and improving long-term quality of life. In this study, we found that neoadjuvant chemotherapy can also be used to downstage the axilla, with nodal conversion in $40 \%$ of patients, a finding that is in keeping with previous studies showing pCR rates of $35 \%-63 \% .{ }^{24}$ This raises the question of surgical axillary staging after neoadjuvant chemotherapy and the potential for limiting ALND to patients who do not achieve a nodal pCR.

Our results challenge conventional thought around chemosensitivity in breast cancer. Specifically, to our knowledge, this study is 1 of only 2 studies ${ }^{25}$ to show a high rate of axillary pCR in patients with ER-positive, PR-negative, HER2-negative disease. In our study, 13 $(43 \%)$ of 30 patients in this subgroup achieved an axillary pCR, which compares favourably with the response seen in patients with TNBC, a group that is preferentially offered neoadjuvant chemotherapy given the high chance of exceptional response. ${ }^{15,18}$ Boland and colleagues ${ }^{25}$ showed that a negative progesterone receptor status has a strong influence on rates of both breast and axillary response to neoadjuvant treatment. This may have farreaching consequences for ER-positive/PR-negative patients, who may not otherwise be considered for neoadjuvant systemic treatment.

In the present study, ER-positive/PR-positive patients were the least likely to achieve an axillary pCR; however, there may still be a role for neoadjuvant treatment in this population, even if only a partial response within the breast is achieved. New evidence shows a survival benefit with systemic treatment in the adjuvant setting in patients with residual invasive disease after neoadjuvant treatment. The CREATE-X (Capecitabine for Residual Cancer as Adjuvant Therapy) trial showed a significant survival benefit with capecitabine in patients with residual invasive disease after neoadjuvant chemotherapy. ${ }^{26}$ This benefit was most pronounced in patients with TNBC, who are at higher risk for both local and distant recurrence. However, Schaefgen and colleagues ${ }^{27}$ failed to find a significant increase in disease-free survival by adding extended 
capecitabine treatment to standard chemotherapy in patients with early TNBC (although only $20 \%$ of patients in the study cohort had undergone neoadjuvant treatment, and the study showed a significant benefit in a subgroup of patients with a nonbasal phenotype). Likewise, the KATHERINE trial showed a significant benefit of trastuzumab-emtansine in HER2-positive patients who did not achieve a pCR after neoadjuvant chemotherapy. ${ }^{28}$ Trastuzumab-emtansine treatment after neoadjuvant therapy is therefore fast becoming the standard of care for HER2-positive patients with residual disease after neoadjuvant chemotherapy. These studies show the added benefit of adjuvant chemotherapy after neoadjuvant treatment for selected groups of partial responders. This will undoubtedly affect guidelines regarding referral for neoadjuvant treatment in the future.

Our study also showed that residual disease within the breast after neoadjuvant treatment is highly predictive of residual disease in the axilla. This seems logical, although some studies have shown a differential response to treatment between the breast and axilla. Morgan and colleagues $^{29}$ found in a retrospective series that $12(14 \%)$ of 83 patients with stage 1-2 breast cancer treated with neoadjuvant chemotherapy had a differential response in the breast and axilla; this is far higher than the rate in our series. Based on these results, we propose the algorithm depicted in Figure 2 going forward and encourage further study to bolster its use.

We also identified variables that were not associated with an axillary node pCR, such as the presence of multiple sonographically abnormal nodes before neoadjuvant chemotherapy. Abnormal nodes detected on ultrasonography are subject to both intra- and interobserver vari- ability, as well as reliance on user proficiency, and can therefore hinder diagnostic accuracy substantially. Studies have been carried out to increase the sensitivity of operator findings, but the evidence is not yet robust enough to support the use of ultrasonography as a predictive or diagnostic tool. ${ }^{27,30,31}$ Although univariate analysis showed tumour grade to be a statistically significant predictor of axillary node response, multivariate analysis failed to confirm this finding. Tumour grade is routinely used to differentiate relatively aggressive cancers from indolent tumours, taking into account the mitotic index, rate of tubular formation and nuclear pleomorphism. One would expect chemotherapy, therefore, to be most active in higher-grade tumours. However, this is not necessarily the case, as the evidence in this area is mixed. ${ }^{32,33}$ Thus, caution should be exercised when taking tumour grade into consideration when counselling patients on the expected effects of chemotherapy.

\section{Limitations}

The main strength of our study is the moderately large cohort of node-positive patients undergoing axillary lymph node dissection after neoadjuvant chemotherapy, which allowed for a granular assessment of predictors of axillary pCR. Nonetheless, the study was limited by the singlecentre, retrospective design, and further study is needed to confirm our results. A multicentre prospective study looking at long-term oncologic outcomes in which nodepositive, ER-positive/PR-negative patients with a complete clinical axillary response are randomly allocated to either ALND or SLNB would provide the most generalizable and definitive validation of our findings.

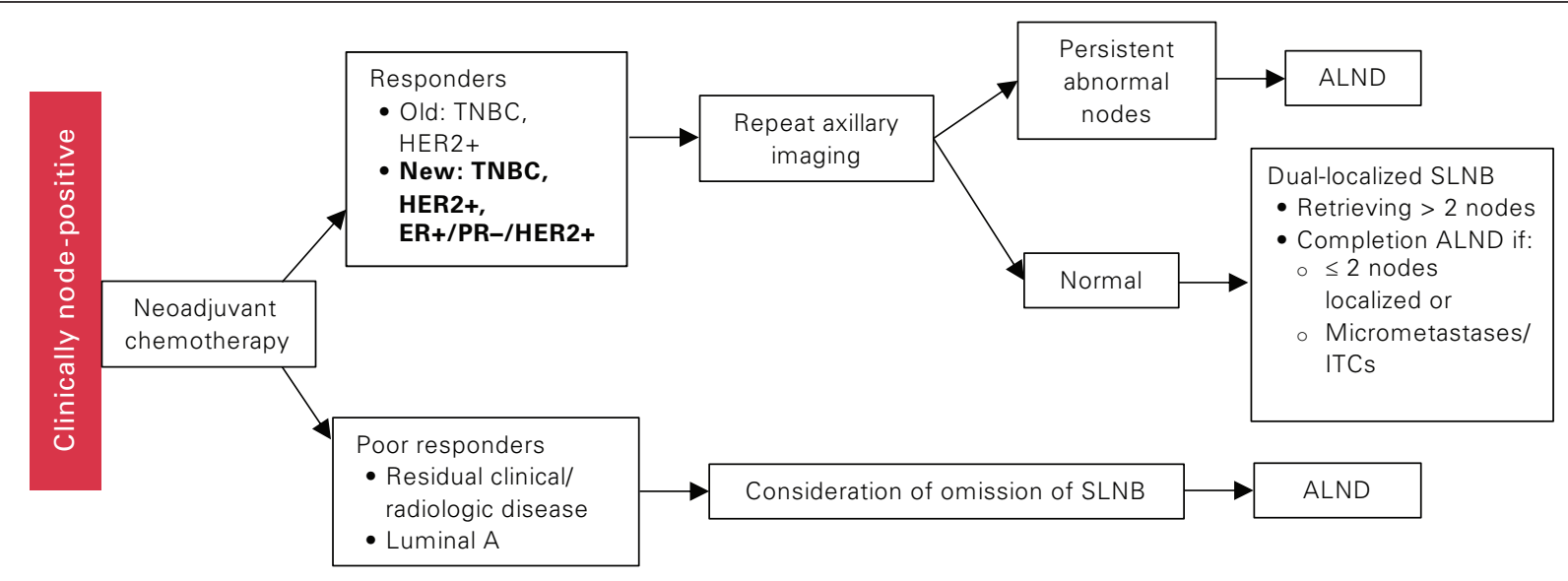

Tumour factors not felt to influence decision-making:

non-high-grade, multiple sonographically abnormal nodes

Fig. 2. Proposed algorithm for treating patients with clinically node-positive breast cancer. ALND = axillary lymph node dissection; ER = estrogen receptor; HER2 = human epidermal growth factor receptor 2; ITC = isolated tumour cell; PR = progesterone receptor; $\mathrm{SLNB}=$ sentinel lymph node biopsy; TNBC $=$ triple-negative breast cancer. 
The NRG Oncology/NSABP B-51/RTOG 1304 trial $^{34}$ and the Comparison of Axillary Lymph Node Dissection With Axillary Radiation for Patients With Node-Positive Breast Cancer Treated With Chemotherapy study, sponsored by the Alliance for Clinical Trials in Oncology (ClinicalTrials.gov Identifier: NCT01901094) are currently evaluating the long-term safety of omitting ALND (in favour of locoregional radiotherapy) in clinically nodepositive patients who achieve an axillary pCR or have residual invasive disease on SLNB. Since the falsenegative rate after SLNB following neoadjuvant chemotherapy has been shown to range from $4.9 \%$ to $14.2 \%,{ }^{11-13,35}$ these trials will determine whether extending radiation therapy as an alternative to ALND in clinically nodepositive patients further supports the avoidance of ALND in this group of patients.

\section{Conclusion}

Our study shows that the absence of residual disease in the breast, and potentially HER2-positive and TNBC biomarker subtypes, can be predictors of an axillary pCR. Our findings also show a potential role for the ER-positive/PRnegative receptor subtype in predicting a complete axillary response, a group of patients previously not felt to respond well to neoadjuvant chemotherapy. Although future study is needed, our findings could help endorse routine SLNB for ER-positive/PR-negative patients who are initially node-positive but become clinically node-negative after neoadjuvant chemotherapy.

Affiliations: From the Division of General Surgery, Department of Surgery, University of Alberta, Edmonton, Alta. (Ladak, Chua, Lesniak, Yakimetz, Rajaee, Olson, Peiris); the Alberta Health Services-Cross Cancer Control Institute, Department of Medical Oncology, University of Alberta, Edmonton, Alta. (Ghosh); the Department of Mathematical and Statistical Sciences, University of Alberta, Alta. (Ghosh); and the Department of Radiation Oncology, Cross Cancer Institute, Edmonton, Alta. (Wiebe, Ghosh).

\section{Competing interests: None declared.}

Contributors: F. Ladak, D. Lesniak, E. Wiebe, D. Olson and L. Peiris designed the study. F. Ladak and N. Chua acquired the data, which F. Ladak, N. Chua, D. Lesniak, S. Ghosh, E. Wiebe, W. Yakimetz, N. Rajaee and L. Peiris analyzed. F. Ladak, S. Ghosh, E. Wiebe and L. Peiris wrote the manuscript, which all authors critically revised. All authors gave final approval of the article to be published.

Content licence: This is an Open Access article distributed in accordance with the terms of the Creative Commons Attribution (CC BYNC-ND 4.0) licence, which permits use, distribution and reproduction in any medium, provided that the original publication is properly cited, the use is noncommercial (i.e., research or educational use), and no modifications or adaptations are made. See: https://creativecommons. org/licenses/by-nc-nd/4.0/.

\section{References}

1. Jatoi I, Hilsenbeck SG, Clark GM, et al. Significance of axillary lymph node metastasis in primary breast cancer. 7 Clin Oncol 1999; 17:2334-40.
2. Yiangou C, Shousha S, Sinnett HD. Primary tumour characteristics and axillary lymph node status in breast cancer. Br 7 Cancer 1999;80: 1974-8.

3. Albertini JJ, Lyman GH, Cox C, et al. Lymphatic mapping and sentinel node biopsy in the patient with breast cancer. FAMA 1996;276: 1818-22.

4. Giuliano AE, Kirgan DM, Guenther JM, et al. Lymphatic mapping and sentinel lymphadenectomy for breast cancer. Ann Surg 1994;220: 391-8.

5. Goyal A, Newcombe RG, Chhabra A, et al.; ALMANAC Trialists Group. Factors affecting failed localisation and false-negative rates of sentinel node biopsy in breast cancer - results of the ALMANAC validation phase. Breast Cancer Res Treat 2006;99:203-8.

6. Krag DN, Anderson SJ, Julian TB, et al. Sentinel-lymph-node resection compared with conventional axillary-lymph-node dissection in clinically node-negative patients with breast cancer: overall survival findings from the NSABP B-32 randomised phase 3 trial. Lancet Oncol 2010;11:927-33.

7. White RL Jr, Wilke LG. Update on the NSABP and ACOSOG breast cancer sentinel node trials. Am Surg 2004;70:420-4.

8. Krag DN, Anderson SJ, Julian TB, et al.; National Surgical Adjuvant Breast and Bowel Project. Technical outcomes of sentinel-lymphnode resection and conventional axillary-lymph-node dissection in patients with clinically node-negative breast cancer: results from the NSABP B-32 randomised phase III trial. Lancet Oncol 2007;8:881-8.

9. Veronesi U, Paganelli G, Viale G, et al. A randomized comparison of sentinel-node biopsy with routine axillary dissection in breast cancer. NEngl 7 Med 2003;349:546-53.

10. Carter S, Neuman H, Mamounas EP, et al. Debating the optimal approach to nodal management after pathologic complete response to neoadjuvant chemotherapy in patients with breast cancer. Am Soc Clin Oncol Educ Book 2019;39:42-8.

11. Boughey JC, McCall LM, Ballman KV, et al. Tumor biology correlates with rates of breast-conserving surgery and pathologic complete response after neoadjuvant chemotherapy for breast cancer: findings from the ACOSOG Z1071 (Alliance) prospective multicenter clinical trial. Ann Surg 2014;260:608-14, discussion 614-6.

12. Boileau JF, Poirier B, Basik M, et al. Sentinel node biopsy after neoadjuvant chemotherapy in biopsy-proven node-positive breast cancer: the SN FNAC study. 7 Clin Oncol 2015;33:258-64.

13. Kuehn T, Bauerfeind I, Fehm T, et al. Sentinel-lymph-node biopsy in patients with breast cancer before and after neoadjuvant chemotherapy (SENTINA): a prospective, multicentre cohort study. Lancet Oncol 2013;14:609-18.

14. Caudle AS, Yang WT, Krishnamurthy S, et al. Improved axillary evaluation following neoadjuvant therapy for patients with nodepositive breast cancer using selective evaluation of clipped nodes: implementation of targeted axillary dissection. 7 Clin Oncol 2016;34: 1072-8.

15. Arnaout A, Lee J, Gelmon K, et al. Neoadjuvant therapy for breast cancer: updates and proceedings from the Seventh Annual Meeting of the Canadian Consortium for Locally Advanced Breast Cancer. Curr Oncol 2018;25:e490-8.

16. van der Hage JA, van de Velde CJH, Julien JP, et al. Preoperative chemotherapy in primary operable breast cancer: results from the European Organization for Research and Treatment of Cancer trial 10902. 7 Clin Oncol 2001;19:4224-37.

17. Wolmark N, Wang J, Mamounas E, et al. Preoperative chemotherapy in patients with operable breast cancer: nine-year results from National Surgical Adjuvant Breast and Bowel Project B-18. 7 Natl Cancer Inst Monogr 2001;30:96-102.

18. Barrio AV, Mamtani A, Edelweiss M, et al. How often is treatment effect identified in axillary nodes with a pathologic complete response after neoadjuvant chemotherapy? Ann Surg Oncol 2016;23:3475-80.

19. Budd DC, Cochran RC, Sturtz DL, et al. Surgical morbidity after mastectomy operations. Am F Surg 1978;135:218-20. 
20. Helms G, Kühn T, Moser L, et al. Shoulder-arm morbidity in patients with sentinel node biopsy and complete axillary dissection data from a prospective randomised trial. Eur 7 Surg Oncol 2009;35: 696-701.

21. Amin MB, Edge SB, Greene FL, et al., editors. AFCC cancer staging manual. 8th ed. Chicago: American College of Surgeons; 2018.

22. DeSnyder SM, Mittendorf EA, Le-Petross C, et al. Prospective feasibility trial of sentinel lymph node biopsy in the setting of inflammatory breast cancer. Clin Breast Cancer 2018;18:e73-7.

23. Systemic therapy for early breast cancer - quick reference guide. Clinical Practice Guideline BR-014 - Version 6. Alberta Health Services; updated 2021 April. Available: https://www.albertahealthservices.ca/ assets/info/hp/cancer/if-hp-cancer-guide-systemic-therapy-early-breast. pdf (accessed 2022 Jan. 18).

24. Pilewskie M, Morrow M. Axillary nodal management following neoadjuvant chemotherapy: a review. 7AMA Oncol 2017;3:549-55.

25. Boland MR, Gilroy D, Nugent T, et al. Impact of progesterone receptor status on response to neoadjuvant chemotherapy in oestrogen receptor positive breast cancer patients. Eur 7 Surg Oncol 2019; 45:878.

26. Masuda N, Lee SJ, Ohtani S, et al. Adjuvant capecitabine for breast cancer after preoperative chemotherapy. $N$ Engl $\mathcal{F}$ Med 2017;376: 2147-59.

27. Schaefgen B, Mati M, Sinn HP, et al. Can routine imaging after neoadjuvant chemotherapy in breast cancer predict pathologic complete response? Ann Surg Oncol 2016;23:789-95.

28. von Minckwitz G, Huang CS, Mano MS, et al.; KATHERINE Investigators. Trastuzumab emtansine for residual invasive HER2positive breast cancer. $N$ Engl 7 Med 2019;380:617-28.
29. Morgan C, Stringfellow TD, Rolph R, et al. Neoadjuvant chemotherapy in patients with breast cancer: Does response in the breast predict axillary node response? Eur 7 Surg Oncol 2020;46:522-6.

30. Kim WH, Kim HJ, Park HY, et al. Axillary pathologic complete response to neoadjuvant chemotherapy in clinically node-positive breast cancer patients: a predictive model integrating the imaging characteristics of ultrasound restaging with known clinicopathologic characteristics. Ultrasound Med Biol 2019;45:702-9.

31. Keune JD, Jeffe DB, Schootman M, et al. Accuracy of ultrasonography and mammography in predicting pathologic response after neoadjuvant chemotherapy for breast cancer. Am 7 Surg 2010;199: 477-84.

32. Kantor O, Sipsy LMN, Yao K, et al. A predictive model for axillary node pathologic complete response after neoadjuvant chemotherapy for breast cancer. Ann Surg Oncol 2018;25:1304-11.

33. Sasanpour P, Sandoughdaran S, Mosavi-Jarrahi A, et al. Predictors of pathological complete response to neoadjuvant chemotherapy in Iranian breast cancer patients. Asian Pac 7 Cancer Prev 2018;19:2423-7.

34. Mamounas EP, Bandos H, White JR, et al. NRG Oncology/NSABP B-51/RTOG 1304: Phase III trial to determine if chest wall and regional nodal radiotherapy (CWRNRT) post mastectomy $(\mathrm{Mx})$ or the addition of RNRT to whole breast RT post breast-conserving surgery (BCS) reduces invasive breast cancer recurrence-free interval (IBCR-FI) in patients (pts) with pathologically positive axillary (PPAx) nodes who are ypN0 after neoadjuvant chemotherapy (NC) [abstract]. F Clin Oncol 2019;37(15 suppl). doi: 10.1200/JCO.2019.37. 15_suppl.TPS600.

35. Cavalcante FP, Millen EC, Zerwes FP, et al. Role of axillary surgery after neoadjuvant chemotherapy. FCO Glob Oncol 2020;6:238-41. 